\title{
The Role of Novel Biomarker in Early Prediction of Preeclampsia in Pregnant Rats
}

\author{
Manar E Selim ${ }^{1,2 *}$, Nouf G Elshmry ${ }^{3}$ and El Himaidi A Rashed ${ }^{1}$ \\ ${ }^{1}$ Department of Zoology, College of Science, King Saud University, Riyadh-11451, KSA, Saudi Arabia \\ ${ }^{2}$ Department of Zoology, Ain Shams University, Cairo, Egypt \\ ${ }^{3}$ Department of Zoology, College of Science, Hail University, Hail, Saudi Arabia
}

\begin{abstract}
Preeclampsia has been known to be implicated in almost $20 \%$ of pregnancy-related maternal deaths and is also known to be the leading cause of a mandated preterm delivery. Pre-eclampsia is a medical condition associated with significant amounts of protein in urine in pregnant ladies reported with hypertension. As this is not a normal situation, the need for development of relevant biomarkers, rapid improvement in current diagnosis and the advancements in treatment is greatly required. The Cellular microparticles are either shed or secreted from cell membranes as endocytic vesicles which are termed as exosomes. In our investigation, evidence of accumulating exosomes in preeclamptic rats that modulate the antigen presentation, and are also capable of suppressing the immune response by exposing NkG2D-ligands has been investigated. The probability of such research on these vesicles and their putative clinical relevance are quite exciting and promising for the future upcoming research. The faster the disease is detected and confirmed with the help of such vesicles, the better would the maternal and fetal prognosis be diagnosed.
\end{abstract}

Keywords: Preeclampsia; Exosomes; NkG2D-ligands; Placenta

\section{Introduction}

The condition pre-eclampsia in pregnant women remains to be important known cause of maternal and prenatal death. Many cases are known to be caused by a shallow implanted placenta that loses its proper functioning, ultimately leading to a medical condition termed Pre-eclampsia Syndrome, resulting in severe immune reaction involved with inflammatory mediators of placenta that acts on the vascular endothelium.

As the maternal parent lacks the receptors responsible for the proteins in certain cases of pre-eclampsia, the placenta results in down regulation of the maternal immune system's response to it. This view is also in accordance with some evidence relating many miscarriages with immunological disorder where the mother's immune system "provokes a devastating attack on the developing child tissues [1]. In addition to this, the perfusion reduction has noted to be secondary in comparison with maternal vessels failed remodeling resulting in deficiency of the supplying the intervillous space to cause preeclampsia [2]. The possibility to identify the evidence of decreased placental perfusion was high in those mother who had restricted growth in babies which was totally not associated with preeclampsia [3]. Failed placental vascular remodeling were further associated with preterm birth which turned to be a pathological evidence [4]. Thus various factors like genetic, constitutional, environmental, physiological and behavioural factors were associated with decreased placental perfusion leading ultimately to preeclampsia.

The previous factors that were leading to the maternal syndrome which may cause cardiovascular disease in further life. Similarly, preeclampsia was the linkage between decreased perfusion and the maternal syndrome. Many so called "toxins" that derived from placenta were proposed, including the cytokines [5] antiangiogenic factors, [6] syncytiotrophoblast microparticles (STBM) [7] and the formed blood products were activated in the intervillous space [8]. Oxidative stress was part of the linkage [9]. The reduced perfusion of the placenta have the capacity to generate certain harmful molecules like reactive oxygen species with consequent activation of monocytes and neutrophils [8] passing through the intervillous space. Oxidative stress will ultimately stimulate the release of cytokines, microparticles, antiangiogenic factors and other such potential linkers that would also be mediated by the same oxidative stress [9]. Unleashes of STBM and exosomes promotes endothelial and immune cell dysfunction, inflammation and necrosis/apoptosis and is somehow associated with the placental pathophysiology in preeclampsia [10-12]. The human hemochorial pregnancy, is the most complicated mode of reproduction that puts a great strain to the maternal organism and creates an immunologic tolerance to the fetal allograft that supports its growth. The placenta is the key for mammalian reproduction success that is known to mediate hormonal, oxygen support and nutrition to the fetus along with secretion of various signaling substances and other immunoregulatory factors which modulate/alter the immune response of the maternal parent during the pregnancy. Many authors [13-18] have shown that the main cell type in the human placenta, the syncytiotrophoblast, releases nanometer-sized microvesicles called exosomes, which are used in the fetal-maternal cross-talk for adaptation of the maternal organism to the ongoing pregnancy. Exosomes are usually 30 to $100 \mathrm{~nm}$-sized microvesicles that have a defined morphology along with phenotype and are formed and released through the late endosomal compartment of a variety of cells [19] with inclusion of syncytiotrophoblast (STB) of human placenta $[12,14]$. To target the cells and transfer the infectious agents between the cells the exosomes have the capacity to carry mRNA, cytosolic and membrane bound proteins, bioactive lipids, as

${ }^{*}$ Corresponding author: Manar E Selim, Department of Zoology, College of Science, King Saud University, Saudi Arabia, Riyadh-11451, KSA, Tel: +966 (0) 56 3588453; E-mail: manar.selim@hotmail.com

Received September 04, 2012; Accepted January 28, 2013; Published January 30,2013

Citation: Selim ME, Elshmry NG, Rashed ElHA (2013) The Role of Nove Biomarker in Early Prediction of Preeclampsia in Pregnant Rats. J Blood Disorders Transf 3:135. doi:10.4172/2155-9864.1000135

Copyright: ( 2013 Selim ME, et al. This is an open-access article distributed unde the terms of the Creative Commons Attribution License, which permits unrestricted use, distribution, and reproduction in any medium, provided the original author and source are credited. 
such. Based on the cell type and the origin of the cell type, exosomes are known to play their role in serving as novel form for the intracellular communication. Dendritic cell originated exosomes have immuneactivating capacity [20], intestinal epithelia and tumors acquired exosomes have a suppressive effect on the immune responses [21]. Thus, human placenta derived exosomes proven by us and others look to be another mechanism that promotes the survival of fetal allograft $[13,15$ 17]. The study reports for the first time that expressing exosomes by the placenta is a new form of storage of intracellular NKG2D ligand and thus contribute to the immune escape of the fetus. Authors including us have shown that placenta releases variety of molecules like immunesuppressive carrying molecules like NKG2D ligands, exosome which suppress immune system of maternal parent $[13,17,18,22]$. The study will focus on the placenta-derived exosomes and discuss their role in preeclampsia.

\section{Materials and Methods}

The current study was carried out on 50 female Wistar rats supplied by Medical College animal house at King Khalid University Hospital (King Saud University). Their average weight was 200-250 g. They were 12-16 weeks old. They were housed in a controlled environment and get free access to water ad labitum. Two or three cycling female rats were housed with a male for 24 hours. The presence of sperms in vaginal smears was considered as day 1 of pregnancy. Rats were divided into two groups (25 rats each) according to the following experimental design:

Group I: Included pregnant rats that were received saline solution $(0.5 \mathrm{ml} / 100 \mathrm{~g}$ body weight) subcutaneously daily starting from day 7 to day 14 of gestation and served as control group.

Group II: Included pregnant rats that were dissolved in sterile solution in the sequential dosage of $10 \mathrm{mg} / 0.5 \mathrm{ml} / 100 \mathrm{~g}$ body weight subcutaneously and simultaneously treated with L-NAME (L-arginine methyl ester) every day starting from the day 7 to day 14 of gestation as mentioned for group I, to make an animal model of preeclampsia [23]. On day 14 of gestation (i.e. 7 days after saline injection in group I or after L-NAME treatment in group II), Placental samples from normal and early preeclamptic rats were isolated following the procedure as described [24] for the following parameters:

\section{Short-term cultures of explants obtained from both early normal and treated placenta}

Short-term placental explants cultures were developed and established similarly as previously mentioned [17]. In short, tiny pieces of chorion villi of around 5-10 $\mathrm{mg}$ of the wet weight were later cultured by using RPMI 1640 medium along with supplementation by $0.5 \%$ BSA (Sigma-Aldrich) or $10 \%$ FCS which was by then ultra centrifuged for $5 \mathrm{~h}$ at $110,000 \mathrm{~g}$. This obtained culture was now strictly maintained at optimum temperature i.e., $37^{\circ} \mathrm{C}$ in $5 \% \mathrm{CO} 2$ and with humidified air. The supernatents obtained were harvested after $24 \mathrm{~h}$ of culture and used for exosome isolation.

\section{Isolation of exosomes from Placental explant culture supernatents}

Supernatents from the culture after clearing the cell debris were collected after $24 \mathrm{~h}$ by sequential centrifugations at 4000 _xg for $30 \mathrm{~min}$ along with 17,000 $\mathrm{xg}$ for $25 \mathrm{~min}$. The pellet obtained was discarded and the supernatant was filtered by a 0.2-_m filter. This was then followed by the ultracentrifugation of the the supernatant at $110,000 \_x g$ for $1 \mathrm{~h}$. Once again the pellet obtained was washed with sterile-filtered
PBS, and again repeated the ultra centrifugation step. Exosome now prepared for electron microscopic observation. For the experiments of NKG2D down-regulation additional purification by $20 \%$ and $40 \%$ sucrose gradient ultracentrifugation was performed and later washed with filtered PBS as mentioned. Preservation of isolated exosomes was done until use at $4^{\circ} \mathrm{C}$ in Phosphate Buffer Saline which was supplemented along with protease inhibitor cocktail (Complete Mini; Roche Diagnostics).

\section{FACS-Analysis}

Anti-NKG2D ligand antibody and matched control isotype antibody were used and exosomes were revealed by FITC-coupled antiCD63 monoclonal antibody performed according to the manufacturer's instructions. After blocking and washing step, 30-min incubation with FITC-conjugated anti-CD63 antibody was analyzed on FACS (BD Biosciences) using Cell Quest software.

\section{TUNEL assay and apoptotic index}

Explants fixed with Para formaldehyde were cut into serial small sections and embedded in paraffin. To assess the apoptosis of placental explants, fluorescence TUNEL assay was performed following the manufacturer's instructions (In Situ Cell Death Detection kit; Roche Molecular Biochemicals, Laval, QC, Canada). The obtained de paraffinised, rehydrated and de waxed sections pretreatment was performed using $20 \mu \mathrm{g} / \mathrm{ml}$ Proteinase K (Sigma-Aldrich) in $10 \mathrm{mmol} / \mathrm{L}$ Trish- $\mathrm{HCl}$ for 15 mins which was then followed by blocking with $10 \%$ normal goat serum and stained the same for TUNEL using FlouresceinUTP containing reaction mixture. In brief, de paraffinised, de waxed, and rehydrated sections were pretreated with $20 \mu \mathrm{g} / \mathrm{ml}$ proteinase $\mathrm{K}$ (Sigma-Aldrich) in $10 \mathrm{mmol} / \mathrm{L}$ Tris- $\mathrm{HCl}$ for 15 minutes, blocked with $10 \%$ normal goat serum, and then stained for TUNEL using a reaction mixture containing fluorescein-dUTP. All those explants sections were blinded and observed with a Leica inverted microscope (Leica Microsystems, Heidelberg, Germany) using a $\times 20$ objective lens. There are three fields that contain a minimum of 900 nuclei which were randomly selected from each section, and digital images were taken of the TUNEL-stained (green) and 4,6-diaminodino-2-phenylindole (DAPI)-stained (blue) sections and were captured and deconvoluted using Slidebook 4.1 software (Intelligent Imaging Innovations Inc., Denver, CO). TUNEL-positive nuclei (apoptotic) and DAPI-stained (total) nuclei were simultaneously counted at a magnification of $400 \times$.

The apoptotic index were calculated in each and every section as the percentage of TUNEL-positive nuclei divided by the total number of DAPI-stained nuclei.

\section{Statistical analysis}

Student's t-tests were used when only two groups were compared. Data are presented as the mean $\pm \mathrm{SE}$, and were considered significant at $P<0.05$.

\section{Results}

\section{Morphology and composition of placental exosomes}

An illustration of the morphology and size of placental exosomes isolated from explant cultures is given in figures 1 and 2. As it can be seen that they have morphological characterization of exosomes obtained from electron micrograph which showed approximately 80 $90 \mathrm{~nm}$ of size, typically cup-saucer shape with electron translucent in control group (Figure 1) whereas ranging from 60-90 nm size (Figure 2 ), typically cup-saucer, spherical with typically less electron translucent and increased electron dense were found in preeclamptic group. 


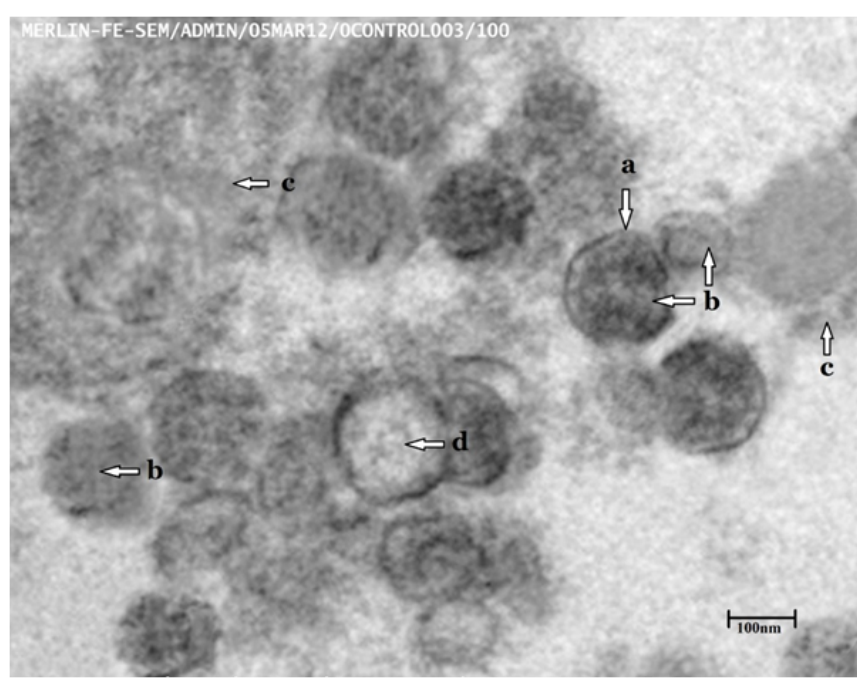

Figure 1: Control group

Size: $80-90 \mathrm{~nm}(\mathrm{a})$

Shape: Typically cup and saucer (b)

Exosomal vesicles: Present (moderate) (c)

Electron translucent: Present (d)

Exosome collection speed pellet at $110,000 \mathrm{~g}$

Sedimentation with sucrose gradient $(0.25-2.5 \mathrm{M})$

Scale bar $100 \mathrm{~nm}$.

The 80 - to $90-\mathrm{nm}$-sized microvesicles displaying the typical cup or saucer shaped morphology of exosomes with moderate and translucent appearance in control group.

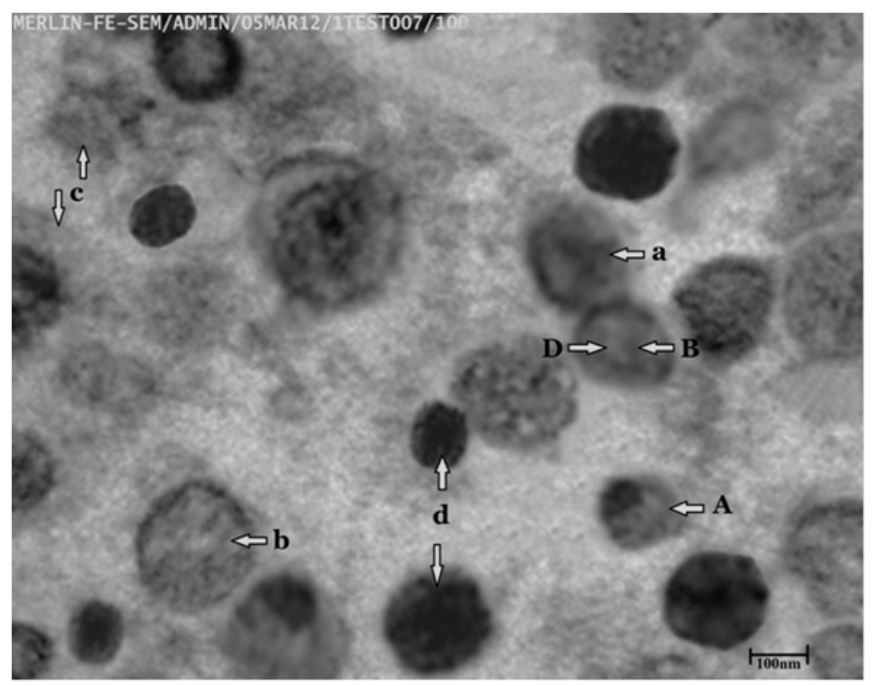

Figure 2: Pre eclamptic group

Size: 60-90nm (60 (A) 90 (a))

Shape: Typically cup and saucer (B), where as $10 \%$ were spherical (b)

Exosomal vesicles: Present (less) (c)

40\% Electron translucent (D), 60\% electron dense (non-translucent) (d)

Exosome collection speed pellet at 110,000

Sedimentation with sucrose gradient $(0.25-2.5 \mathrm{M})$

Scale bar $100 \mathrm{~nm}$.

The 60- to90-nm-sized microvesicles displaying the typical cup saucer and spherical -shaped morphology of increased exosomes with dense and translucent appearance in preeclamptic group

\section{Explants from early placenta release NKG2D ligand-bearing exosomes}

Isolated exosomes were captured on anti NKG2D ligand and revealed by anti-CD63 in our electron microscopy and flow cytometric analysis (Figures 1-3). The results indicate that the placenta releases exosomes expressing NKG2D ligands on the surface.

\section{TUNEL assay and apoptotic index}

To determine whether placental explants were undergoing apoptosis, the placental sections from control and treated groups were stained with TUNEL. As shown in figure 4 no significant TUNEL-positive signal was detectable in tissue from control rats. However, preeclamptic rats resulted in TUNEL-positive staining. Explants from early trimester treated rats with L-NAME demonstrated increased placental apoptosis as compared to control, as determined by a TUNEL assay and index, which may be blocked by the presence of NKG2D ligand. Placental explants from control group did not show significant apoptosis (\% negligible). Compared with the apoptotic index of untreated control explants of early pregnancy, a subsequent treatment with L-NAME resulted in increase in the apoptotic index $(P<0.05$; Figure 5$) 37 \%$ of cells were found to be underwent apoptosis in preeclampsia (White arrow in Figure 4). The apoptotic index from control to treated explants increased significantly 0.2 to $37 \%$ respectively $(P<0.05)$.

\section{Discussion}

Pregnancy is not the sole condition gaining from studies of placental exosomes. Understanding how the well-being of the fetal allograft is created can benefit transplantation. Moreover, to establish a nutrient supply and to evade the host immune response many invasive, proliferative and immune tolerance mechanisms that support the normal human pregnancy have been exploited by preeclampsia. Thus, release of exosomes that can edit immune responses to promote survival and well-being of the fetus during preeclampsia, might be one common link between the physiological state of pregnancy and the pathological state of preeclampsia. Taking the methodological consideration described earlier, there are few investigations into isolated pregnancy-specific exosomes so far. They comprise studies of exosomes isolated from the peripheral blood of pregnant women performed by the group of Taylor et al. [15] and studies of exosomes isolated from ex vivo placental explant cultures from first trimester normal pregnancy performed by another groups $[13,17,22]$. Knowledge, gained from exosome research in reproduction, could lead to identification of novel diagnostic and therapeutic approaches in preeclampsia. Intercellular communication caused by microvesicles MV has opened new perspectives in understanding the role of the placenta as the one of the most powerful regulatory organ that uses MV-mediated signaling to target and also reprogram maternal cells for the reproduction benefits. Microvesicular body MVB/late endosomes are major protein sorting in the cellular endocytic pathway. These vesicles are stored, sorted for protein degradation to lysosomes, or released as exosomes into the extracellular milieu. Few mechanisms still remain unknown such as the cellular mechanisms for sorting of proteins for secretion, storage, or degradation [25]. In some cells, MVB has an additional role of serving as temporary protein storage compartments [26]. In our investigation the increased level of placental exosomes as seen in preeclamptic group but not in control group revealed a novel mechanism detecting frequently in early rather than in late onset pre-eclampsia. The presence of NKG2D ligands on placental exosomes in situ was evidenced by previous phenotypic analyses of the microvesicles that were isolated from the supernatant of placental explant cultures [27]. Our electron 

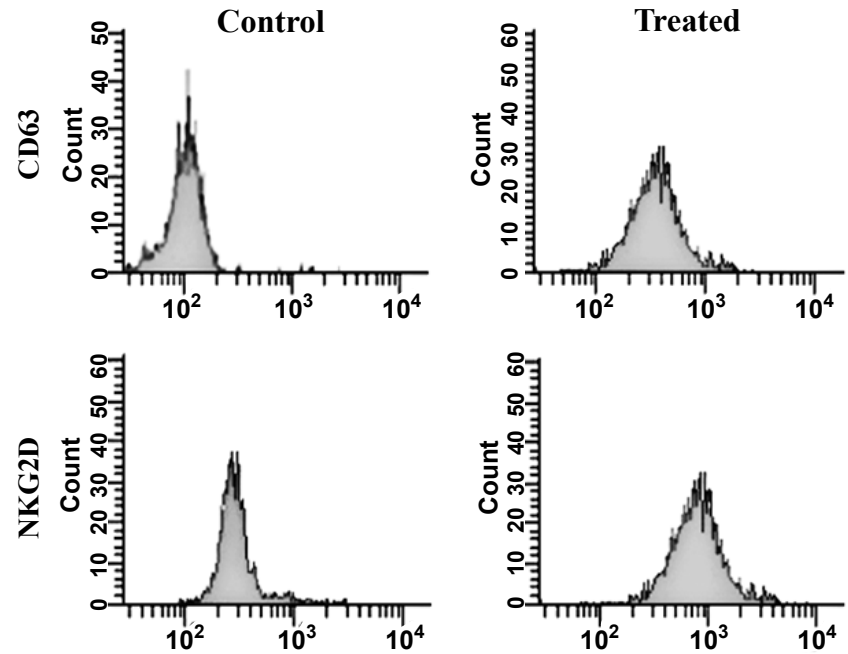

Figure 3: Flow cytometric analyses indicates that the secreted NKG2D ligandtagged vesicle like structures revealed expression of known exosomal marker such as CD63, verifying their exosomal nature.

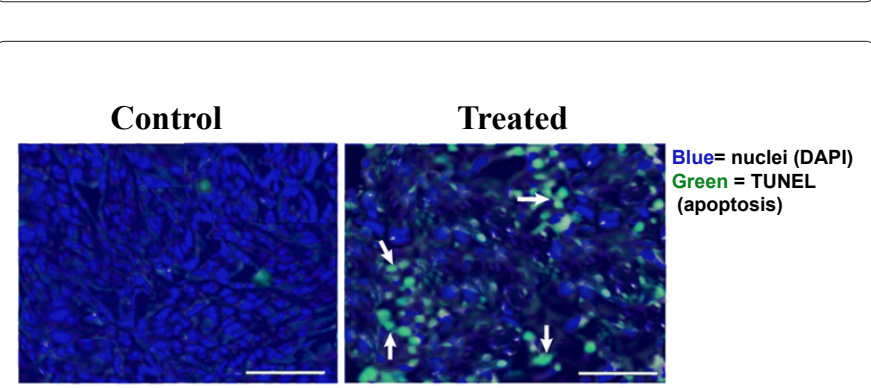

Figure 4: The level of placental extracts apoptosis, which was assessed using the TUNEL method, was negligible in control conditions as compared to increased level in preeclamptic group. TUNEL-positive apoptotic cells were visualized with FITC (green) and cell nuclei were stained with DAPI (blue). Scale bars: $100 \mu \mathrm{m}$.

microscopic study (Figures 1 and 2) and FACS analyses (Figure 3) showed that the secreted NKG2D ligand-loaded microvesicles displayed morphology, size, and expression of known exosomal markers such as CD63 confirming their exosomal nature. Tumor-shed soluble NKG2D ligands have been shown to impair the cytotoxic antitumor response by NKG2D receptor down-regulation [28]. Soluble form of the ligands is generated by the mechanism Protease-mediated cleavage of NKG2D ligands on the cell membrane [29]. The release of placental exosomes, which has the capacity to mimic cell-cell interactions, represents an alternative mechanism for generation of soluble bioactive NKG2D ligands were observed in tumor cell lines [30,31]. Studies on Exosome involve both immunosuppressive and immunostimulatory role [22,32]. Apoptosis of activated T cells were induced by exosome-associated secretion of FasL from STB and thus promoted the fetal allograft survival $[13,14]$. However, exosomal FasL is not the sole mediator of immune escape of the fetus.The release of placental NKG2D ligand bearing exosomes provide a platform for permanent engagement of the same NKG2D. Recent studies have shown that this engagement not only impairs the NKG2D function but includes suppression of other, perforin-independent cytotoxicity pathways [33], confirming the finding of Taylor et al. [15] that placental exosomes suppress the CD3-_ chain and JAK3 expression in T lymphocytes. Increased cytokines are seen in the circulation of preeclamptic women reasons being still unknown [34] Multiple studies demonstrated that increased inflammatory cytokine production leads to endothelial dysfunction, increased placenta apoptosis and other complications like kidney abnormalities that are relevant to the pathophysiology of preeclampsia PE [35]. Although there are animal models of PE available, but little of them have delineated the cause of increased exosomes during this mechanism. Here, using a novel biomarker for early detection of PE in pregnant rats, we demonstrate that the production of exosomes through this mechanism and expression characteristics of the NKG2D ligand may play an important role in the fetal allograft survival as represented by the percentage of apoptosis (Figure 5). Our result goes with subsequent studies, elevated circulating STBM were detected more frequently in early rather than in late onset pre-eclampsia [36]. Increased levels were not found in normotensive pregnancies as would be associated with isolated deficient placentation [36]. The syncytial surface of the placenta renews itself, as do other biological surfaces, by apoptosis which is controlled cell fragmentation with release of subcellular microparticles in forms that are easy to clear by phagocytic systems [37]. Several forms of trophoblast debris [38], formed in this way, can be detected in the maternal circulation, including soluble cytokeratin of placental origin, soluble fetal DNA and soluble fetal RNA and exosomes. In addition, all such factors are increased in the circulation in pre-eclamptic women. Trophoblast hypoxia induces apoptosis [39] and significantly more apoptosis can be detected in situ in samples taken from the hypoxic placentas of pre-eclamptic women [40].

We highlighted here in that exosomes comprise a stimulus to the systemic inflammatory response in pre-eclamptic pregnancies. The current evidence that exosomes from pre-eclamptic placenta differ in quality from those from normal placentas differ with [41]. In addition, in this study the entire population of exosomes was studied with reference bearing NKG2D ligand together with their placental source. The mild features of preeclampsia and rescue of preeclampsia-like features by recombinant exosomes bearing NKG2D ligand suggest its regulatory role in programming the severity of preeclampsia manifested by apoptosis and appoptic index (Figures 4 and 5). Thus, our model provides potential opportunities to study the influence of exosomes and bearing NKG2D ligands and their contribution to preeclampsia.

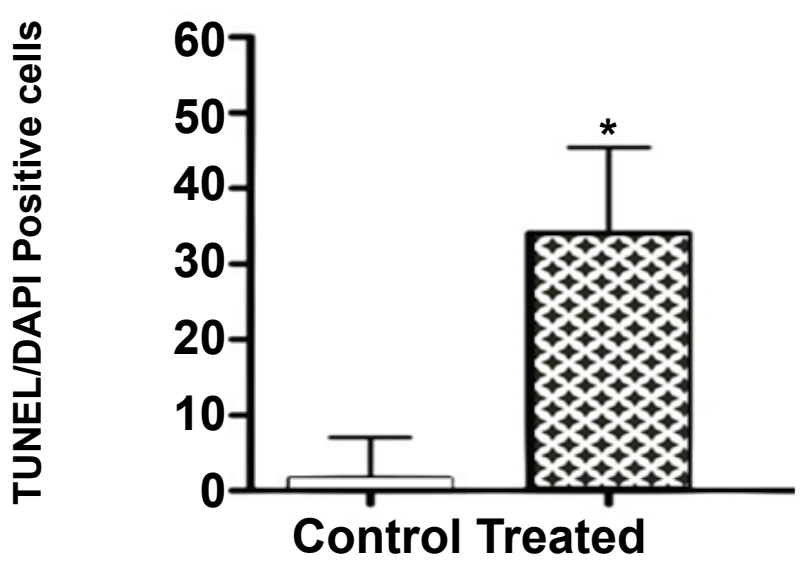

Figure 5: Percentage of TUNEL-positive nuclei in control versus treated groups. 
More such analyses need to be performed to come to a consensus that the role of exosomes in preeclampsia and related diseases, recurrent abortions and infertility waits to be evaluated. Understanding mechanisms of apoptosis and other variants of programmed cell death, at the molecular level provides a deeper platform in acquiring knowledge into the disease processes and may thus influence in framing up of much advanced therapeutic strategy.

\section{References}

1. Sargent IL, Germain SJ, Sacks GP, Kumar S, Redman CW (2003) Trophoblas deportation and the maternal inflammatory response in pre-eclampsia. J Reprod Immunol 59: 153-160

2. Roberts J (2007) Pre-eclampsia a two-stage disorder: What is the linkage? Are there directed fetal/placental signals? In: FL, MB, editors. Pre-eclampsia: Etiology and clinical practice. New York: Cambridge University Press pp. 183194.

3. Khong TY, De Wolf F, Robertson WB, Brosens I.(1986) Inadequate maternal vascular response to placentation in pregnancies complicated by preeclampsia and by small-for-gestational age infants. British Journal of Obstetrics and Gynaecology 93:1049-1059.

4. Arias F, Rodriquez L, Rayne SC, Kraus FT (1993) Maternal placenta vasculopathy and infection: two distinct subgroups among patients with preterm labor and preterm ruptured membranes. Am J Obstet Gynecol 168: 585-591.

5. Schipper EJ, Bolte AC, Schalkwijk CG, Van Geijn HP, Dekker GA (2005) TNFreceptor levels in preeclampsia--results of a longitudinal study in high-risk women. J Matern Fetal Neonatal Med 18: 283-287.

6. Maynard SE, Venkatesha S, Thadhani R, Karumanchi SA (2005) Soluble Fms-like tyrosine kinase 1 and endothelial dysfunction in the pathogenesis of preeclampsia. Pediatr Res 57: 1R-7R.

7. Redman CW, Sargent IL (2005) Latest advances in understanding preeclampsia. Science 308:1592-1594.

8. Mellembakken JR, Aukrust P, Olafsen MK, Ueland T, Hestdal K, et al. (2002) Activation of leukocytes during the uteroplacental passage in preeclampsia. Hypertension 39: 155-160.

9. Roberts JM, Hubel CA (1999) Is oxidative stress the link in the two-stage mode of pre-eclampsia? Lancet 354: 788-789.

10. Guller S (2009) Role of the syncytium in placenta-mediated complications of preeclampsia. Thromb Res 124: 389-392.

11. Toth B, Lok CA, Böing A, Diamant M, van der Post JA, et al. (2007) Microparticles and exosomes: impact on normal and complicated pregnancy. Am J Reprod Immunol 58: 389-402.

12. Redman CW, Sargent IL (2007) Microparticles and immunomodulation in pregnancy and pre-eclampsia. J Reprod Immunol 76: 61-67.

13. Abrahams VM, Straszewski-Chavez SL, Guller S, Mor G (2004) First trimester trophoblast cells secrete Fas ligand which induces immune cell apoptosis. Mo Hum Reprod 10: 55-63.

14. Frängsmyr L, Baranov V, Nagaeva O, Stendahl U, Kjellberg L, et al. (2005) Cytoplasmic microvesicular form of Fas ligand in human early placenta: switching the tissue immune privilege hypothesis from cellular to vesicular level. Mol Hum Reprod 11: 35-41.

15. Taylor DD, Akyol S, Gercel-Taylor C (2006) Pregnancy-associated exosomes and their modulation of T cell signaling. J Immunol 176: 1534-1542.

16. Sabapatha A, Gercel-Taylor C, Taylor DD (2006) Specific isolation of placenta-derived exosomes from the circulation of pregnant women and their immunoregulatory consequences. Am J Reprod Immunol 56: 345-355.

17. Mincheva-Nilsson L, Nagaeva O, Chen T, Stendahl U, Antsiferova J et al.(2006) Placenta-derived soluble MHC class I chain-related molecules down-regulate NKG2D receptor on peripheral blood mononuclear cells during human pregnancy: a possible novel immune escape mechanism for fetal survival. $J$ Immunol 176:3585-3592.

18. Hedlund M, Stenqvist AC, Nagaeva O, Kjellberg L, Wulff M, et al. (2009) Human placenta expresses and secretes NKG2D ligands via exosomes that downmodulate the cognate receptor expression: evidence for immunosuppressive function. J Immunol 183: 340-351.
19. Théry C, Zitvogel L, Amigorena S (2002) Exosomes: composition, biogenesis and function. Nat Rev Immunol 2: 569-579.

20. Andre F, Escudier B, Angevin E, Tursz T, Zitvogel L (2004) Exosomes for cancer immunotherapy. Ann Oncol 15 Suppl 4: iv141-144.

21. Karlsson M, Lundin S, Dahlgren U, Kahu H, Pettersson I, et al. (2001) "Tolerosomes" are produced by intestinal epithelial cells. Eur J Immunol 31: 2892-2900.

22. Frangsmyr L, Baranov V, Nagaeva O, Stendahl U, Kjellberg L, MinchevaNilsson L (2005) Cytoplasmic microvesicular form of Fas ligand in human early placenta:switching the tissue immune privilege hypothesis from cellular to vesicular level. Molecular Human Reproduction 11:35-41.

23. Curtis NE, Gude NM, King RG, Marriott PJ, Rook TJ et al. (1995) Nitric oxide in normal human pregnancy and preeclampsia. Hypertens Pregn 14: 339-349.

24. Stenqvist AC, Chen T, Hedlund M, Dimova T, Nagaeva O, et al. (2008) An efficient optimized method for isolation of villous trophoblast cells from human early pregnancy placenta suitable for functional and molecular studies. Am J Reprod Immunol 60: 33-42.

25. de Gassart A, Geminard C, Fevrier B, Raposo G, Vidal M (2003) Lipid raftassociated protein sorting in exosomes. Blood 102: 4336-4344.

26. Jiang L, Erickson A, Rogers J (2002) Multivesicular bodies: a mechanism to package lytic and storage functions in one organelle? Trends Cell Biol 12: 362 367

27. van Niel G, Porto-Carreiro I, Simoes S, Raposo G (2006) Exosomes: a common pathway for a specialized function. J Biochem 140: 13-21.

28. González S, Groh V, Spies T (2006) Immunobiology of human NKG2D and its ligands. Curr Top Microbiol Immunol 298: 121-138.

29. Salih HR, Antropius H, Gieseke F, Lutz SZ, Kanz L, et al. (2003) Functional expression and release of ligands for the activating immunoreceptor NKG2D in leukemia. Blood 102: 1389-1396.

30. Clayton A, Tabi Z (2005) Exosomes and the MICA-NKG2D system in cancer Blood Cells Mol Dis 34: 206-213.

31. Clayton A, Mitchell JP, Court J, Linnane S, Mason MD, et al. (2008) Human tumor-derived exosomes down-modulate NKG2D expression. J Immunol 180: 7249-7258.

32. Li XB, Zhang ZR, Schluesener HJ, Xu SQ (2006) Role of exosomes in immune regulation. J Cell Mol Med 10: 364-375.

33. Coudert JD, Scarpellino L, Gros F, Vivier E, Held W (2008) Sustained NKG2D engagement induces cross-tolerance of multiple distinct NK cell activation pathways. Blood 111: 3571-3578.

34. Saito S, Shiozaki A, Nakashima A, Sakai M, Sasaki Y (2007) The role of the immune system in preeclampsia. Mol Aspects Med 28: 192-209.

35. Sargent IL, Borzychowski AM, Redman CW (2007) NK cells and pre-eclampsia J Reprod Immunol 76: 40-44

36. Goswami D, Tannetta DS, Magee LA, Fuchisawa A, Redman CW, et al. (2006) Excess syncytiotrophoblast microparticle shedding is a feature of early-onse pre-eclampsia, but not normotensive intrauterine growth restriction. Placenta 27: 56-61.

37. Huppertz B, Frank HG, Kingdom JC, Reister F, Kaufmann P (1998) Villous cytotrophoblast regulation of the syncytial apoptotic cascade in the human placenta. Histochem Cell Biol 110: 495-508.

38. Redman CW, Sargent IL (2000) Placental debris, oxidative stress and preeclampsia. Placenta 21: 597-602.

39. Levy R, Smith SD, Chandler K, Sadovsky Y, Nelson DM (2000) Apoptosis in human cultured trophoblasts is enhanced by hypoxia and diminished by epidermal growth factor. Am J Physiol Cell Physiol 278: C982-988.

40. Ishihara N, Matsuo $\mathrm{H}$, Murakoshi $\mathrm{H}$, Laoag-Fernandez JB, Samoto $\mathrm{T}$, et al (2002) Increased apoptosis in the syncytiotrophoblast in human term placentas complicated by either preeclampsia or intrauterine growth retardation. Am J Obstet Gynecol 186: 158-166.

41. Cester N, Staffolani R, Rabini RA, Magnanelli R, Salvolini E, et al. (1994) Pregnancy induced hypertension: a role for peroxidation in microvillus plasma membranes. Mol Cell Biochem 131: 151-155. 